\title{
Numerical Study of MHD Casson Liquid Stream over an Infinite Vertical Porous Plate with Newtonian Heating
}

\section{R.L.V. Renuka Devi ${ }^{1}$, A. Neeraja ${ }^{2}$, Y. Swapna ${ }^{3}$, V. Nagaradhika ${ }^{4}$, P.S.S.R. Sujatha ${ }^{5}$ and M. Krishna Murthy ${ }^{6, *}$}

\author{
1,3Department of Mathematics, Sri Venkateswara University, Tirupati, 517502, A.P., \\ India \\ 2,5 Department of Mathematics, Aditya College of Engineering, Surampalem, East \\ Godavari, 533437, A.P., India \\ ${ }^{4}$ Department of Mathematics, GITAM Deemed to be University, Bengaluru, 562163, \\ Karnataka, India \\ ${ }^{6}$ Department of Mathematics, School of Applied Sciences, REVA University, \\ Bengaluru, 560064, Karnataka, India
}

Article Type: Article

Article Citation: Renuka Devi RLV, Neeraja A, Swapna Y, Nagaradhika V, Sujatha PSSR, Krishna Murthy M. Numerical study of MHD Casson liquid stream over an infinite vertical porous plate with Newtonian heating. Indian Journal of Science and Technology. 2020; 13(02), 149-160. D0l: 10.17485/ ijst/2020/v013i02/147338

Received date: September 7, 2019 Accepted date: September 7, 2019

*Author for correspondence: $M$. Krishna Murthy kalyankrishna51@ gmail.com $\odot$ Department of Mathematics, School of Applied Sciences, REVA University, Bengaluru, Karnataka, India

\begin{abstract}
Objectives: Magnetohydrodynamics (MHD) stream of Casson liquid over a limitless vertical porous plate with Newtonian heating is considered. Casson liquid is non-Newtonian liquid. Methods/ statistical analysis: The governing nonlinear equations are comprehended utilizing shooting system. Findings: The impacts of relevant governing parameters on the liquid velocity and the temperature are exhibited graphically. The coefficient of skin friction and the rate of heat transfer are determined numerically. The present outcomes have been good simultaneousness with existing outcomes under some unique cases. Applications: Porous plates are additionally used in the design of heat exchangers, computer assemblies, polymer industry, and automotive industry.
\end{abstract}

Keywords: MHD, Casson Liquid, Newtonian Heating, Shooting Technique, Vertical Porous Plate.

\section{Nomenclature}
$u^{\prime}$ is the velocity of the fluid in $x^{\prime}$ -direction
$T^{\prime}$ is the fluid temperature
$h$ is the coefficient of heat transfer
$k$ is the thermal conductivity

$\beta_{1}$ is the volume coefficient of thermal expansion.

$B_{0}$ is strength of the magnetic field along $y^{\prime}$-axis.

$\beta$ is the Casson parameter 


$$
\begin{aligned}
& v^{\prime} \text { is the suction velocity } \\
& v \text { is the kinematic viscosity } \\
& T_{\infty}^{\prime} \text { is the free stream temperature } \\
& g \text { is the gravity of acceleration } \\
& \sigma \text { is the electrical conductivity of the } \\
& \text { fluid } \\
& \rho \text { is density of the fluid } \\
& S \text { is the suction parameter } \\
& c_{p} \text { is the specific heat constant } \\
& q_{r} \text { is the radiative heat flux along } y^{\prime} \text {-axis }
\end{aligned}
$$

$G r$ is the thermal Grashof number

$M$ is the magnetic field parameter

$u$ and $\theta$ are the fluid flow velocity and

the temperature respectively

Pr is the Prandtl number

$R$ is the radiation parameter,

$E c$ is the Eckert number.

$\mu$ is the viscosity

\section{Introduction}

Magnetohydrodynamics (MHD) steam of Casson liquid over an infinite vertical porous plate is used in various Industrial and Engineering areas. Casson liquid is one of the non-Newtonian liquid. It is having profoundly viscosity nature. Human blood is one of the instances of Casson liquid. Porous plates are additionally used in the design of heat exchangers, computer assemblies, polymer industry, and automotive industry. The employments of magnetohydrodynamics are cooling of nuclear reactors, controlling boundary layer stream, geothermal energy extraction, sun power vitality gatherers, plasma aerodynamics, etc. The impact of Casson fluid flow over channels was addressed by the researchers [1-9]. In Refs. [10-11], researchers developed MHD natural convection flow past an impulsively started infinite vertical porous plate with Newtonian heating in the presence of radiation and effects of thermal radiation and mass diffusion on free convection flow near a vertical plate with Newtonian heating. The researchers [12-19] discovered the effect of MHD over a various geometries (vertical channel, vertical cylinder, stretching sheet).

The present assessment MHD stream of Casson liquid over an endless vertical permeable plate with Newtonian heating is examined. The governing non-linear differential equations are settled utilizing Ruge-Kutta fourth-order strategy along with shooting system. The impacts of governing parameters such as Prandtl number, Magnetic field parameter, Casson parameter, Suction parameter, and radiation parameter on the liquid velocity and the temperature are exhibited in graphically. The skin friction coefficient and the Nusselt number are found in numerically and showed up in tabular structure.

\section{Mathematical Formulation of the Problem}

Consider the steady hydrodynamic stream of Casson liquid over a limitless vertical plate with Newtonian heating. The plate in the upward way along the $x$-axis and $y$-axis normal to it as showed up in Figure 1. Assumed that the heat transfer rate from the plate with a finite heat capacity is corresponding to the local surface temperature $\left(T^{\prime}\right)$.

The under Boussinesq estimate for the continuity equation, conservation of mass, and the energy equations are as per the following: 


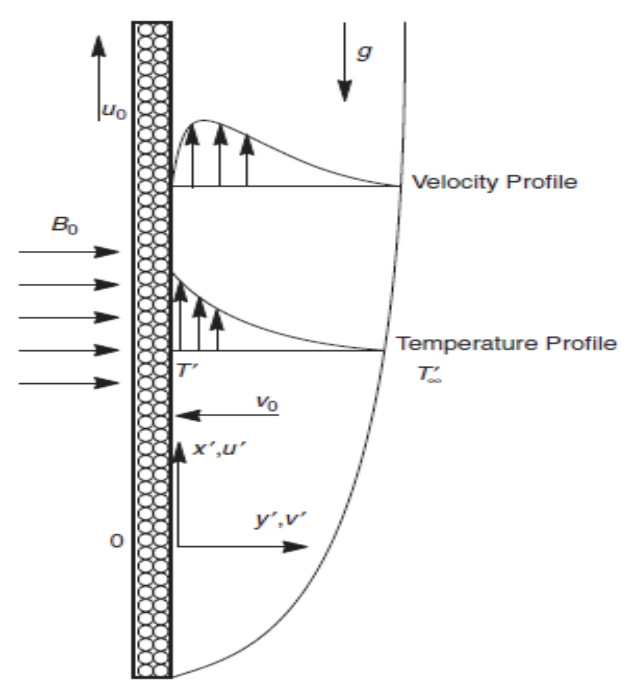

FIGURE 1. Physical model of the problem.

$$
\begin{gathered}
\frac{\partial v^{\prime}}{\partial y^{\prime}}=0 \\
v^{\prime} \frac{\partial u^{\prime}}{\partial y^{\prime}}=v\left(1+\frac{1}{\beta}\right) \frac{\partial^{2} u^{\prime}}{\partial y^{\prime 2}}+g \beta_{1}\left(T^{\prime}-T_{\infty}^{\prime}\right)-\frac{\sigma B_{0}^{2}}{\rho} u^{\prime} \\
v^{\prime} \frac{\partial T^{\prime}}{\partial y^{\prime}}=\frac{k}{\rho c_{p}} \frac{\partial^{2} T^{\prime}}{\partial y^{\prime 2}}-\frac{1}{\rho c_{p}} \frac{\partial q_{r}}{\partial y^{\prime}}+\frac{\mu}{\rho c_{p}}\left(1+\frac{1}{\beta}\right)\left(\frac{\partial u^{\prime}}{\partial y^{\prime}}\right)^{2}+\frac{\sigma B_{0}^{2}}{\rho} u^{\prime 2}
\end{gathered}
$$

The limit conditions are as follows:

$$
\left.\begin{array}{l}
u^{\prime}=u_{0}, \frac{\partial T^{\prime}}{\partial y^{\prime}}=-\frac{h}{k} T^{\prime} \text { at } y^{\prime}=0 \\
u^{\prime} \rightarrow 0, T^{\prime} \rightarrow T_{\infty}^{\prime} \text { as } y^{\prime} \rightarrow \infty
\end{array}\right\}
$$

Integrating equation (1) for suction constant, we have

$$
v^{\prime}=-v_{0}
$$

Where $v_{0}>0$ is the normal velocity of suction at the plate?

Now equations (2) and (3) are as the following:

$$
\begin{gathered}
-v_{0} \frac{\partial u^{\prime}}{\partial y^{\prime}}=v\left(1+\frac{1}{\beta}\right) \frac{\partial^{2} u^{\prime}}{\partial y^{\prime 2}}+g \beta_{1}\left(T^{\prime}-T_{\infty}^{\prime}\right)-\frac{\sigma B_{0}^{2}}{\rho} u^{\prime} \\
-v_{0} \frac{\partial T^{\prime}}{\partial y^{\prime}}=\frac{k}{\rho c_{p}} \frac{\partial^{2} T^{\prime}}{\partial y^{\prime 2}}-\frac{1}{\rho c_{p}} \frac{\partial q_{r}}{\partial y^{\prime}}+\frac{\mu}{\rho c_{p}}\left(1+\frac{1}{\beta}\right)\left(\frac{\partial u^{\prime}}{\partial y^{\prime}}\right)^{2}+\frac{\sigma B_{0}^{2}}{\rho} u^{\prime 2}
\end{gathered}
$$


The radiation heat flux $q_{r}$ in equation (7) fulfills the accompanying non-linear differential equation [7]

$$
\frac{\partial q_{r}}{\partial y^{\prime}}=4 \alpha \sigma^{*}\left(T^{\prime 4}-T_{\infty}^{\prime 4}\right)
$$

where $\alpha$ is the absorption coefficient and $\sigma^{*}$ is the Stefan-Boltzman constant. Assuming that the temperature contrasts inside the stream are adequately little such that $T^{\prime 4} \mathrm{can}$ be expressed as a linear function of $T^{\prime}$ and utilizing the Taylor series expression about $T^{\prime 4}$ in the wake of disregarding higher order terms, we have:

$$
T^{\prime 4} \cong 4 T_{\infty}^{\prime 3} T^{\prime}-3 T_{\infty}^{\prime 4}
$$

From equations (8), (9), and (7) we get

$$
-v_{0} \frac{\partial T^{\prime}}{\partial y^{\prime}}=\frac{k}{\rho c_{p}} \frac{\partial^{2} T^{\prime}}{\partial y^{\prime 2}}-\frac{16 \alpha \sigma^{*} T_{\infty}^{\prime 3}}{\rho c_{p}}\left(T^{\prime}-T_{\infty}^{\prime}\right)+\frac{\mu}{\rho c_{p}}\left(1+\frac{1}{\beta}\right)\left(\frac{\partial u^{\prime}}{\partial y^{\prime}}\right)^{2}+\frac{\sigma B_{0}^{2}}{\rho} u^{\prime 2}
$$

The non-dimensional quantities are as per the following:

$$
\begin{aligned}
& y=\frac{y^{\prime} h}{k}, u=\frac{u^{\prime}}{u_{0}}, \theta=\frac{T^{\prime}-T_{\infty}^{\prime}}{T_{\infty}^{\prime}}, S=\frac{v_{0} k}{h v}, M=\frac{\sigma B_{0}^{2} k^{2}}{\mu h^{2}} \\
& \left.G r=\frac{g \beta_{1} T_{\infty}^{\prime} k^{2}}{v u_{0} h^{2}}, \operatorname{Pr}=\frac{\mu c_{p}}{k}, E c=\frac{\mu u_{0}^{2}}{k T_{\infty}^{\prime}}, R=\frac{16 \alpha \sigma^{*} k T_{\infty}^{\prime 3}}{h^{2}}\right\}
\end{aligned}
$$

Using equation (11), from equations (6), (10), and (4) are as per the following:

$$
\begin{gathered}
\left(1+\frac{1}{\beta}\right) \frac{d^{2} u}{d y^{2}}+S \frac{d u}{d y}+G r \theta-M u=0 \\
\frac{d^{2} \theta}{d y^{2}}+\operatorname{Pr} S \frac{d \theta}{d y}-R \theta+E c\left(1+\frac{1}{\beta}\right)\left(\frac{d u}{d y}\right)^{2}+E c M u^{2}=0
\end{gathered}
$$

The limit conditions are

$$
\left.\begin{array}{l}
u=1, \frac{d \theta}{d y}=-(\theta+1) \text { at } y=0 \\
u \rightarrow 0, \theta \rightarrow 0 \text { as } y \rightarrow \infty
\end{array}\right\}
$$

Physical amounts of intrigue are the skin friction coefficient $C_{f}$ and the local rate of heat transfer coefficient $\mathrm{Nu}$ are characterized as

$$
C_{f}=\frac{\tau^{\prime}}{\rho G r u_{0}^{2}}=-\left.\left(1+\frac{1}{\beta}\right) \frac{d u}{d y}\right|_{y=0} \text { and } N u=-\left.\frac{v}{u_{0}\left(T^{\prime}-T_{\infty}^{\prime}\right)} \frac{d T^{\prime}}{d y^{\prime}}\right|_{y=0}=1+\frac{1}{\theta(0)}
$$




\section{Results and Discussion}

The present assessment reveals that hydromagnetic stream of Casson liquid over a vast vertical porous plate with Newtonian heating. Here we explored the present outcomes for both Newtonian $(\beta \rightarrow \infty)$ and non-Newtonian $(\beta=0.5)$ cases. Here the Casson parameter taken as $\beta=0.5$. The nonlinear governing equations can be unwound utilizing Runge-Kutta fourth-order methodology along with shooting system. The impacts of governing parameters on the liquid velocity and the temperature distribution are appeared in graphically while the skin friction coefficient and the Nusselt number are determined numerically and are showed up in tabular structure. The present outcomes have been incredible simultaneousness with the current examinations under some restricted cases.

The impact of magnetic field parameter $M$ on the liquid flow velocity $u(y)$ is showed up in Figure 2. We saw that the liquid velocity $u(y)$ lessens for higher estimations of magnetic field parameter $M$ further the momentum boundary layer thickness diminishes this causes the Lorentz force. The effect of Suction parameter $S$ on the liquid velocity $u(y)$ and the temperature distribution $\theta(y)$ are appeared in Figures 3 and 4. We saw that the liquid velocity $u(y)$ and the temperature $\theta(y)$ rot with the impact of $S$. This reality that of the impact of the suction is to take away the hot liquid on the vertical plate and consequently diminishing the liquid velocity with lessens free convection currents intensity. The variation on fluid velocity $u(y)$ with the impact of thermal Grashof number $G r$ is shown in Figure 5. We have seen that the liquid velocity $u(y)$ improves with growing

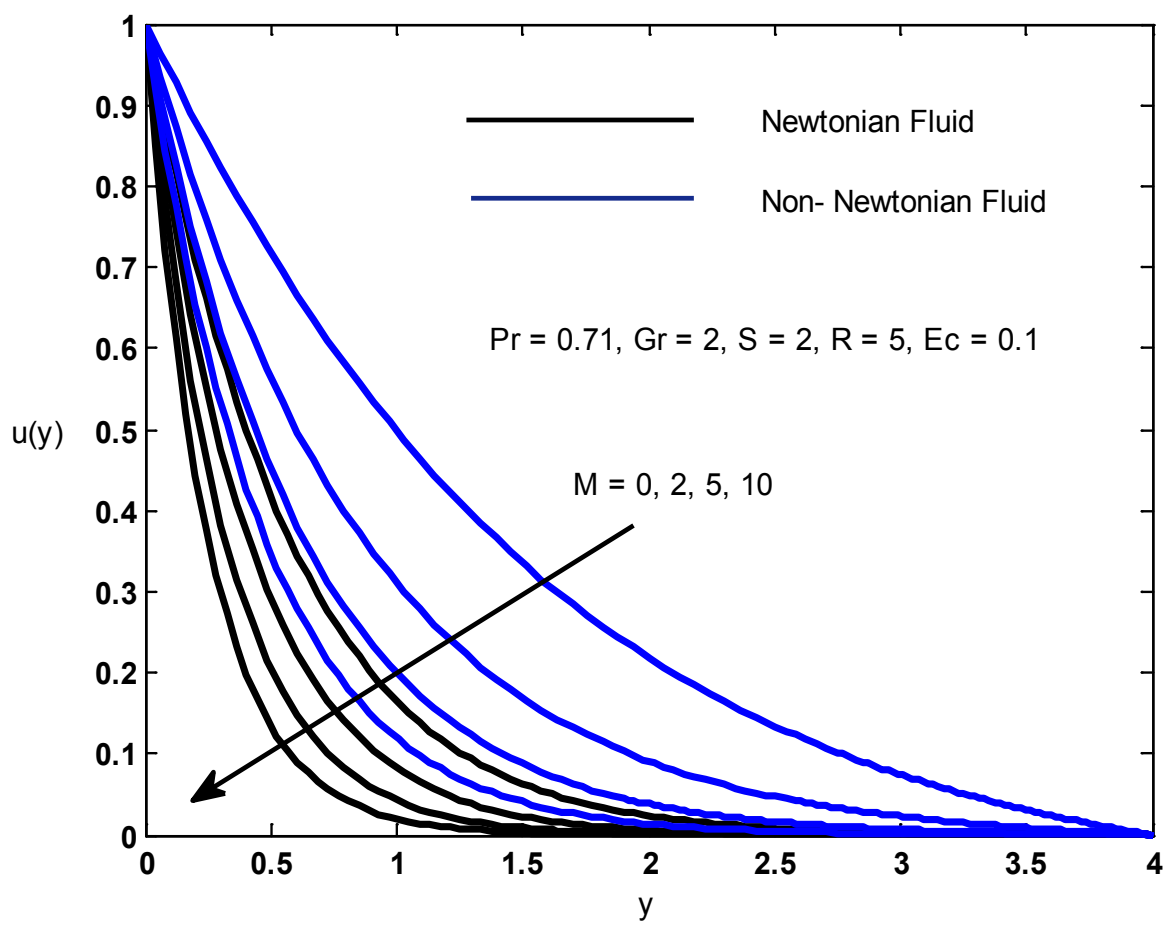

FIGURE 2. The fluid velocity $u(y)$ with $M$. 
Numerical Study of MHD Casson Liquid Stream over an Infinite Vertical Porous Plate with Newtonian Heating

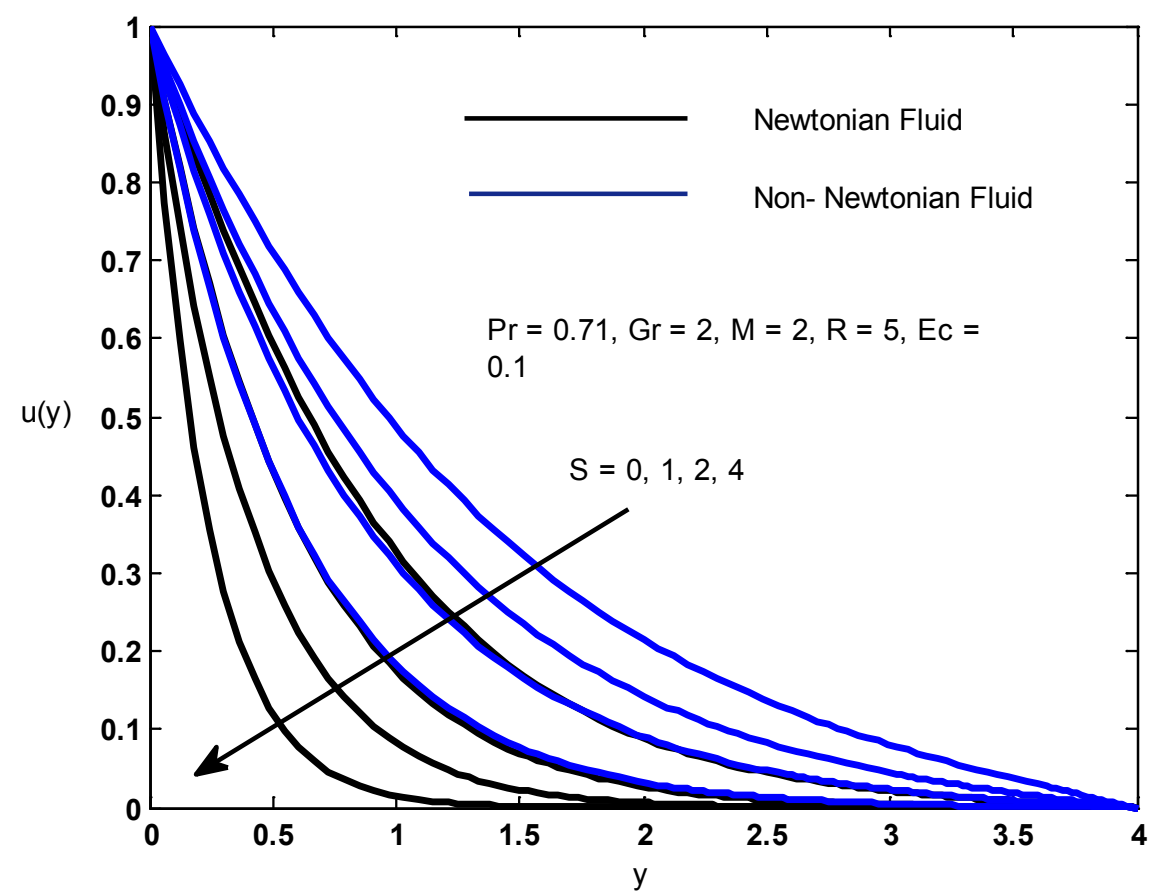

FIGURE 3. The fluid velocity $u(y)$ with $S$.

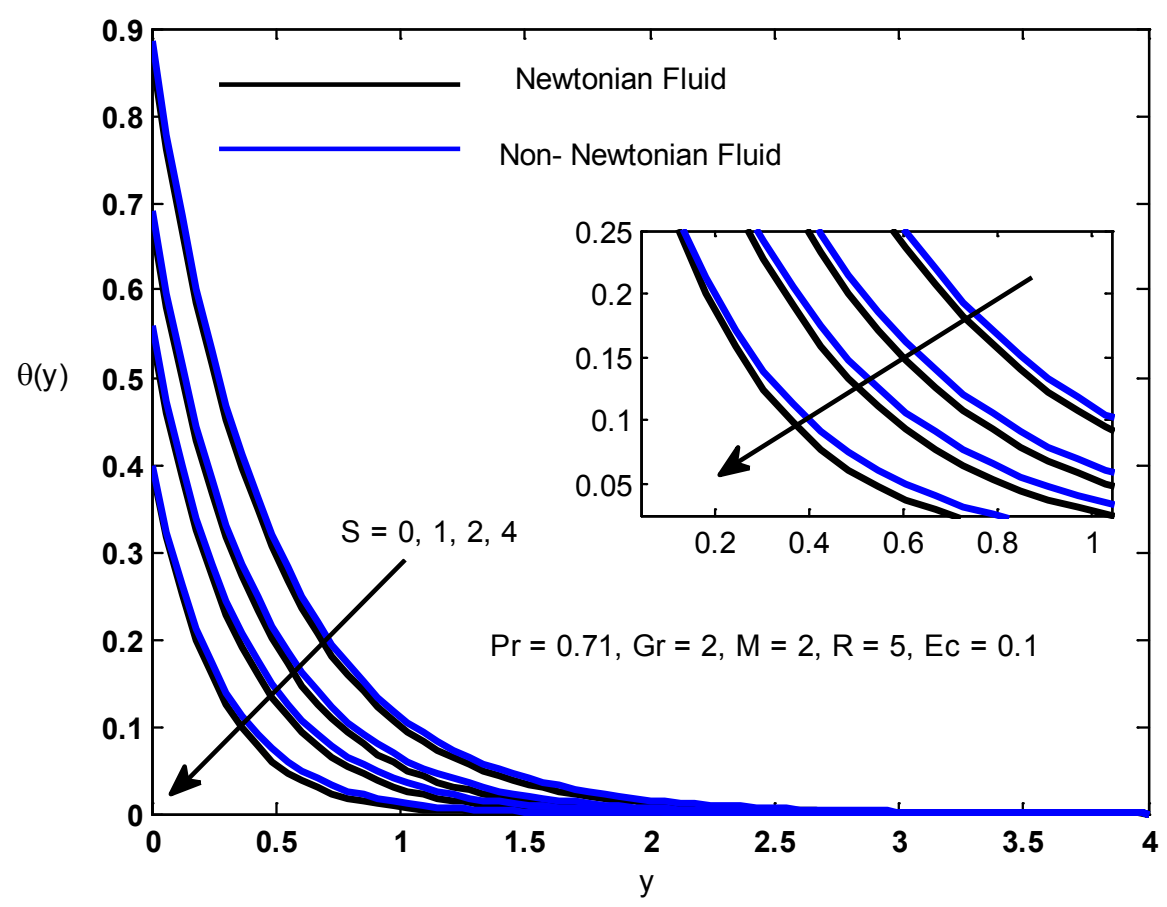

FIGURE 4. The temperature distribution $\theta(y)$ with $S$. 


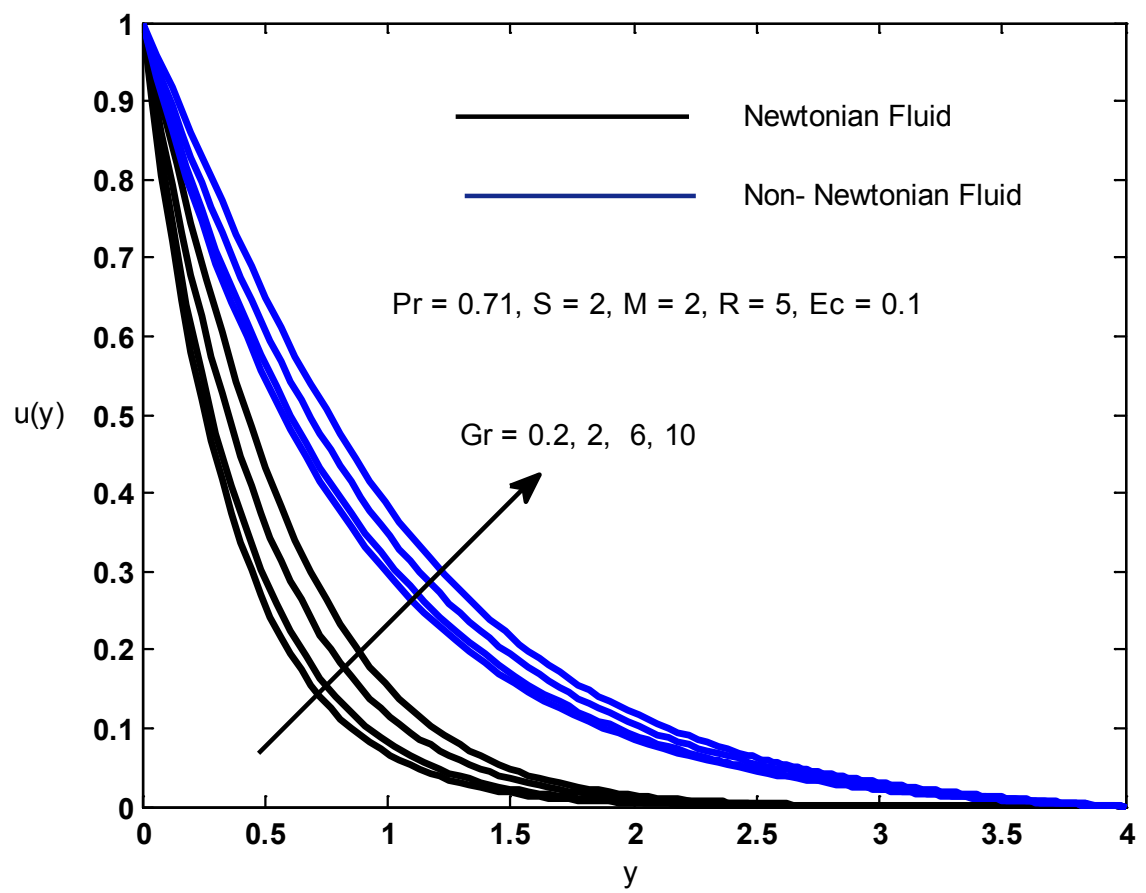

FIGURE 5. The fluid velocity $u(y)$ with $G r$.

Grashof number. This causes the higher estimations of Grashof number then the viscous force impact rots and additions in momentum boundary layer thickness. The impact of Casson parameter $\beta$ on the liquid velocity $u(y)$ is demonstrated in Figure 6 . We saw that the liquid velocity diminishes for higher estimations of Casson parameter. The yield pressure rot which causes the creation for opposition force which make the liquid velocity decreases. The liquid is closer to the Newtonian liquid.

Figure 7 speaks to the impact of Prandtl number Pr on the temperature distribution $\theta(y)$. We uncover that the temperature rots with extending the Prandtl number. This causes that for higher estimations of Prandtl number relate to decreases the thermal diffusivity. Figure 8 portrays the impact of radiation parameter $R$ on the temperature distribution $\theta(y)$. We have seen that the temperature diminishes with a growing radiation parameter which causes for higher estimations of thermal radiation parameter have affinity to absorb the radiative heat and subsequently the buoyancy force rots as well as thermal boundary layer lessens.

The numerical estimations of the skin friction coefficient and the Nusselt number for distinct governing parameters are showed up in Table 1. We saw that the skin friction coefficient overhauls for higher estimations of Prandtl number, magnetic field parameter, suction parameter and radiation parameter and the skin friction coefficient rots with growing Grashof number and Eckert number. The Nusselt number improves for upgrading Prandtl number, Grashof number, radiation parameter and suction parameter and the Nusselt number declines for higher estimations of Eckert number and magnetic 
Numerical Study of MHD Casson Liquid Stream over an Infinite Vertical Porous Plate with Newtonian Heating

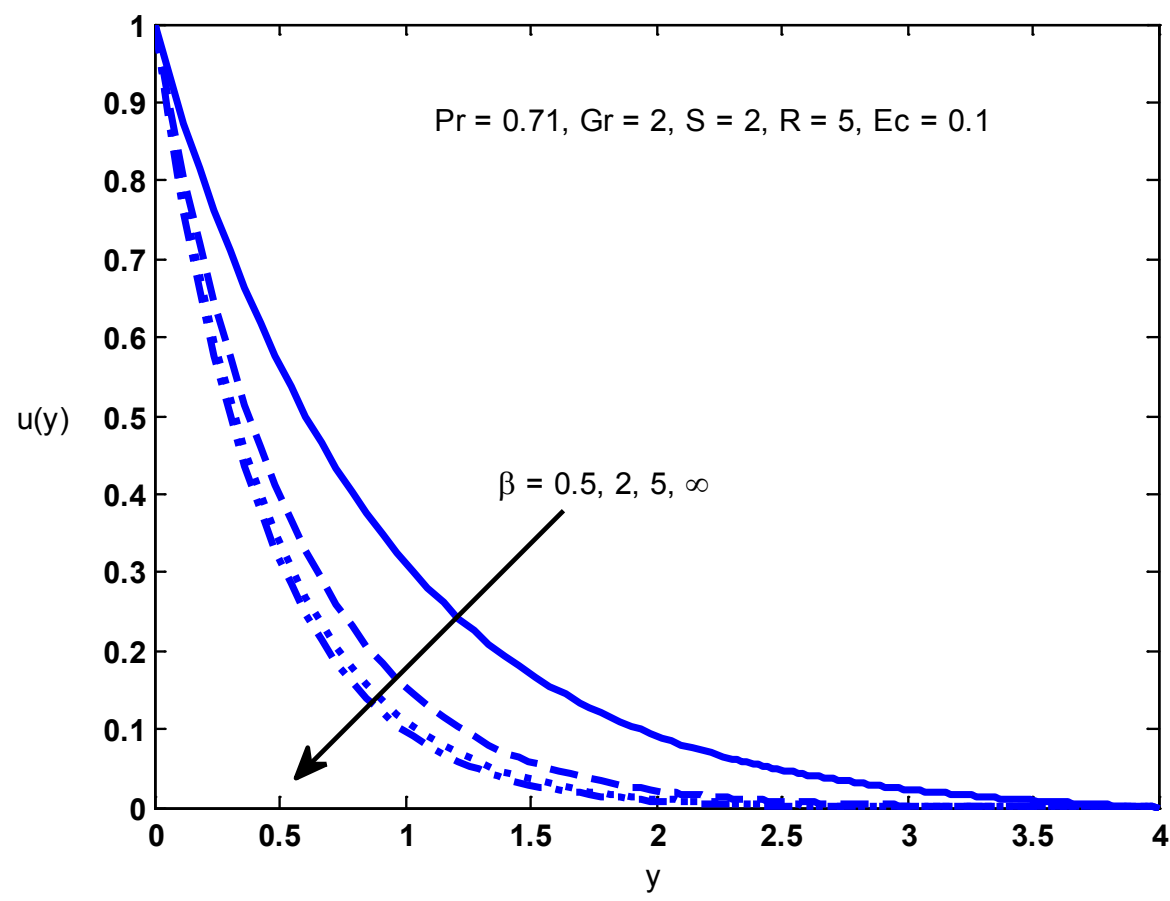

FIGURE 6. The fluid velocity $u(y)$ with $\beta$.

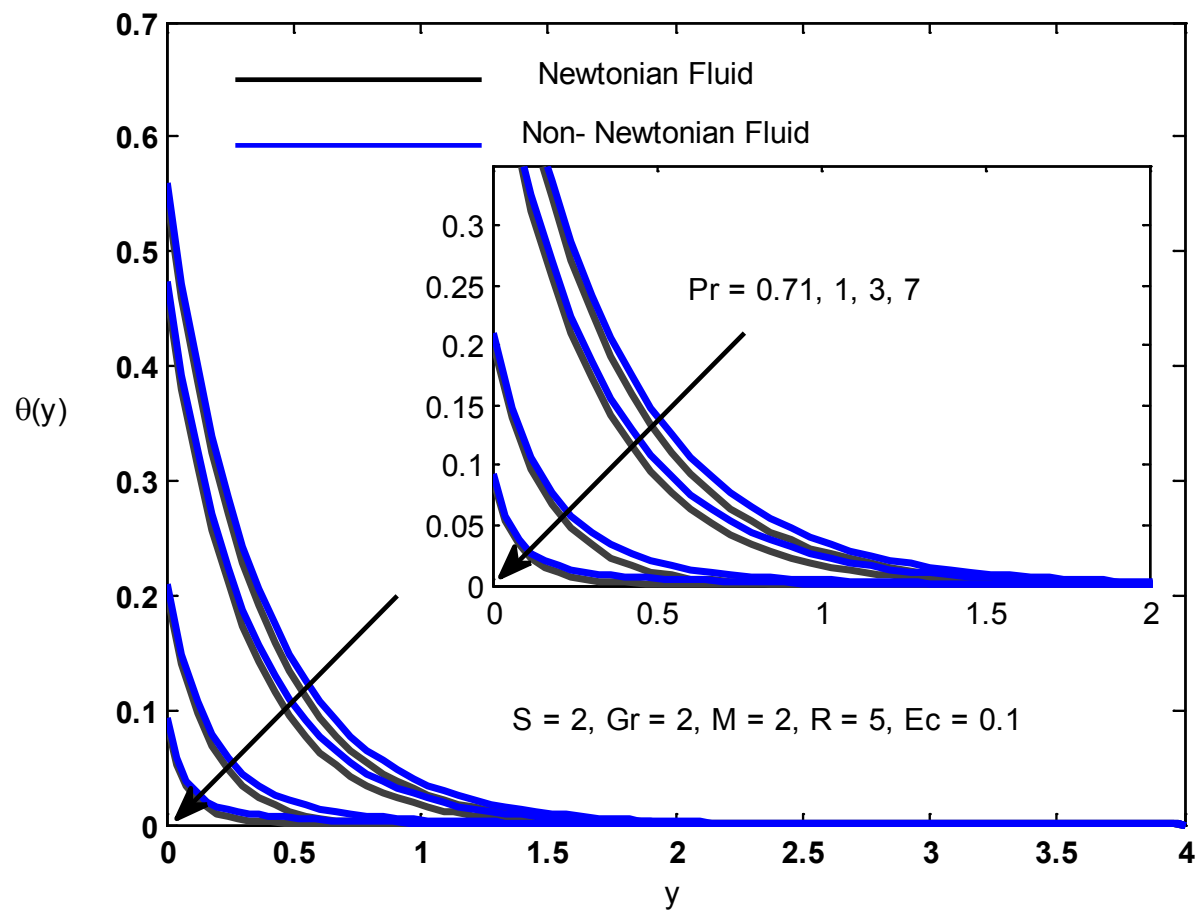

FIGURE 7. The temperature distribution $\theta(y)$ with $\operatorname{Pr}$. 


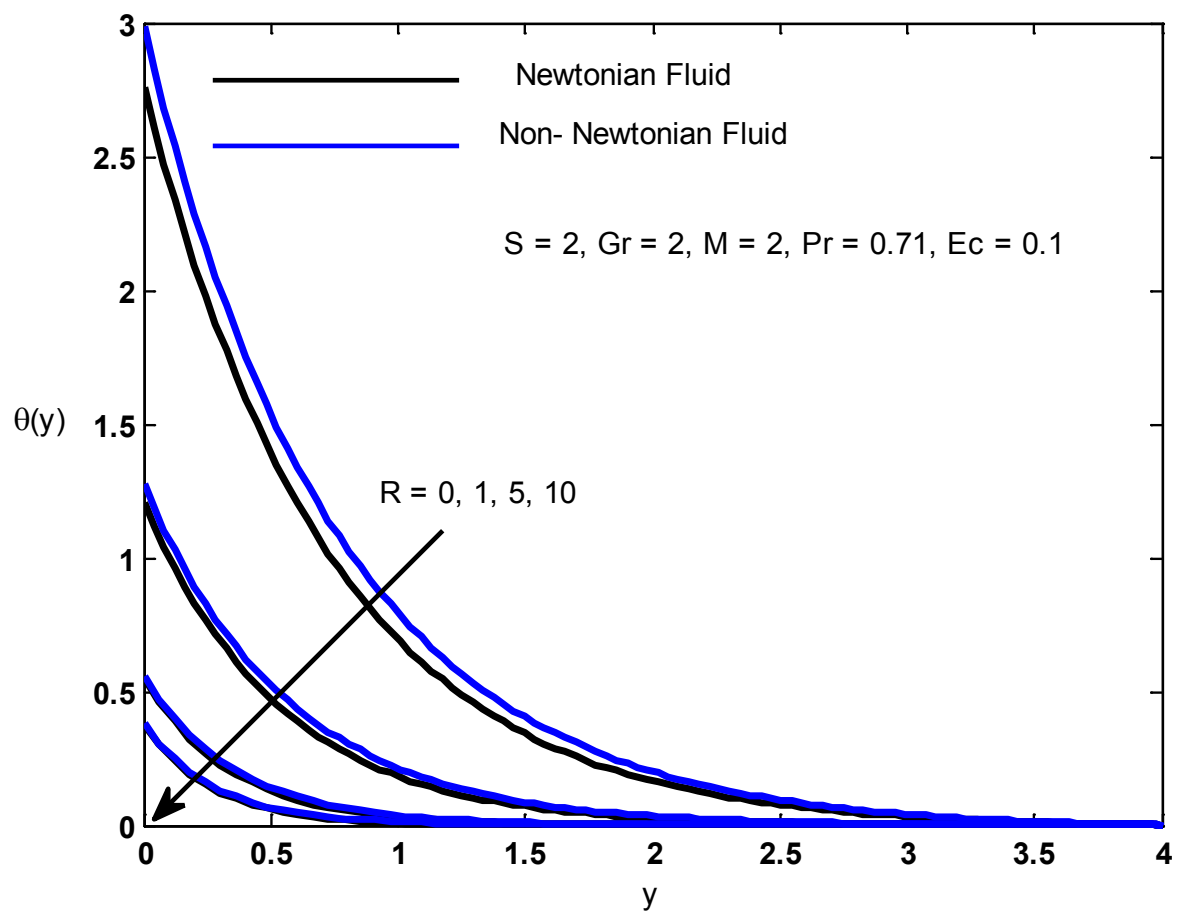

FIGURE 8. The temperature distribution $\theta(y)$ with $R$.

TABLE 1. The numerical values of the skin friction coefficient $C f$ and the rate of heat transfer $N u$ for distinct values of $\operatorname{Pr}, G r, E c, S, M$, and $R$ when the absence of $(\beta \rightarrow \infty)$

\begin{tabular}{|l|l|l|l|l|l|l|l|l|l|}
\hline Pr & Gr & Ec & S & M & \multirow{2}{*}{$\boldsymbol{R}$} & In [10] & $\begin{array}{l}\text { Present } \\
\text { result } \\
(\beta \rightarrow \infty)\end{array}$ & In [10] & $\begin{array}{l}\text { Present } \\
\text { result } \\
(\beta \rightarrow \infty)\end{array}$ \\
\hline 0.3 & 2 & 0.1 & 2 & 2 & 5 & 2.18782 & 2.18782 & 2.40855 & 2.40855 \\
\hline 0.71 & & & & & & 2.31881 & 2.31881 & 2.8436 & 2.8436 \\
\hline 1 & & & & & & 2.38021 & 2.38021 & 3.18447 & 3.18447 \\
\hline 3 & & & & & & 2.43219 & 2.43219 & 6.04361 & 6.04361 \\
\hline 7 & & & & & & 2.44549 & 2.44549 & 12.7096 & 12.7096 \\
\hline 0.71 & 0.2 & & & & & 2.61649 & 2.61649 & 2.82578 & 2.82578 \\
\hline & 6 & & & & & 1.80347 & 1.80347 & 2.86364 & 2.86364 \\
\hline & 10 & & & & & 1.24627 & 1.24627 & 2.88043 & 2.88043 \\
\hline & 15 & & & & & 0.53924 & 0.53924 & 2.88994 & 2.88994 \\
\hline & 2 & 0 & & & & 2.39937 & 2.39937 & 3.05598 & 3.05598 \\
\hline & & 0.05 & & & & 2.38081 & 2.38081 & 2.94103 & 2.94103 \\
\hline & & 0.1 & & & & 2.36233 & 2.36233 & 2.8392 & 2.8392 \\
\hline
\end{tabular}


Numerical Study of MHD Casson Liquid Stream over an Infinite Vertical Porous Plate with Newtonian Heating

\begin{tabular}{|l|l|l|l|l|l|l|l|l|l|}
\hline & & 0.15 & & & & 2.34392 & 2.34392 & 2.74838 & 2.74838 \\
\hline & & 0.2 & & & & 2.3256 & 2.3256 & 2.66687 & 2.66687 \\
\hline & & 0.1 & 0 & & & 0.93886 & 0.93886 & 2.15346 & 2.15346 \\
\hline & & & 1 & & & 1.58837 & 1.58837 & 2.48615 & 2.48615 \\
\hline & & & 4 & & & 3.91856 & 3.91856 & 3.59115 & 3.59115 \\
\hline & & & 6 & & & 5.60228 & 5.60228 & 4.33171 & 4.33171 \\
\hline & & & 2 & 0 & & 1.63506 & 1.63506 & 2.94084 & 2.94084 \\
\hline & & & & 5 & & 2.99838 & 2.99838 & 2.74832 & 2.74832 \\
\hline & & & & 10 & & 3.43956 & 3.43956 & 2.66563 & 2.66563 \\
\hline & & & & 2 & 0 & 0.99746 & 0.99746 & 1.4348 & 1.4348 \\
\hline & & & & & 1 & 1.7479 & 1.7479 & 1.83538 & 1.83538 \\
\hline & & & & & 5 & 2.05239 & 2.05239 & 2.85988 & 2.85988 \\
\hline & & & & & 10 & 2.14835 & 2.14835 & 3.69343 & 3.69343 \\
\hline & & & & & 20 & 2.21501 & 2.21501 & 4.90493 & 4.90493 \\
\hline
\end{tabular}

field parameter. The present outcomes have been great simultaneousness with existing outcomes [6] when the nonattendance of Casson parameter $(\beta \rightarrow \infty)$.

\section{Conclusions}

In this study, we uncover that the hydromagnetic stream of Casson liquid over an infinite vertical porous channel with Newtonian heating is examined. The nonlinear differential equations are grasped utilizing shooting strategy. The present outcomes are contrasted differently in relation to Newtonian and non-Newtonian liquid and are demonstrated graphically. The impact of the suitable governing parameters on the liquid velocity and the temperature are showed up in graphically. The skin friction coefficient and the rate of heat transfer are surveyed numerically and showed up in tabular structure. The destinations of the present outcomes are as follows:

- The momentum boundary layer diminishes with the impact of magnetic parameter. The liquid velocity and the temperature are decay for higher estimations of suction parameter.

- The momentum boundary layer increment for higher estimations of Grashof number and inverse conduct is seen with extending Casson parameter.

- Thermal boundary layer rots for higher estimations of Prandtl number and Radiation parameter. The skin friction coefficient increments with extending the Prandtl number, magnetic field parameter, suction parameter and radiation parameter and the skin friction coefficient diminishes for higher estimations of Grashof number and Eckert number. The Nusselt number improves for upgrading Prandtl number, Grash of 
number, radiation parameter and suction parameter and the Nusselt number decreases for higher estimations of Eckert number and magnetic field parameter.

- The present outcomes have been incredible simultaneousness with existing outcomes [10] when the nonappearance of Casson parameter i.e. $(\beta \rightarrow \infty)$.

\section{References}

1. Muthuraj R, Selvi RK, Srinivas S. Thermodynamic analysis on MHD Casson nanofluid flow in a vertical porous space with stretching walls. Global Journal of Researches in Engineering. 2018; $18(1), 1-15$.

2. Kataria HR, Patel HR. Heat and mass transfer in MHD Casson fluid flow past over an oscillating vertical plate embedded in porous medium with ramped wall temperature. Propulsion and Power Research. 2018; 7(3), 257-267.

3. Vijaya N, Hari Krishna Y, Kalyani K, Reddy GVR. Soret and radiation effects on an unsteady flow of a Casson fluid through porous vertical channel with expansion and contraction. Frontiers in Heat and Mass Transfer. 2018; 11(19), 1-11.

4. Shah Z, Islam S, Ayaz H, Khan S. Radiative heat and mass transfer analysis of micropolar nanofluid flow of Casson fluid between two rotating parallel plates with effects of hall current. Journal of Heat Transfer. 2018; 141(2), 1-34.

5. Biswas R, Ahmed SF. Effects of hall current and chemical reaction on MHD unsteady heat and mass transfer of Casson nanofluid flow through a vertical plate. Journal of Heat Transfer. 2018; 140(9), 1-12.

6. Narahari M, Kamran M. MHD natural convection flow past an impulsively started infinite vertical porous plate with Newtonian heating in the presence of radiation. International Journal of Numerical Methods for Heat \& Fluid Flow. 2016; 26(6), 1932-1953.

7. Narahari M, Dutta B.K. Effects of thermal radiation and mass diffusion on free convection flow near a vertical plate with Newtonian heating. Chemical Engineering Communications. 2012; 199(5), 628-643.

8. Saqib M, Ali F, Khan I, Sheikh NA. Heat and mass transfer phenomena in the flow of Casson fluid over an infinite oscillating plate in the presence of first order chemical reaction and slip effect. Neural Computing Applications. 2018; 30(7), 2159-2172.

9. Khan A, Khan I, Khan A, Shafie S. Heat transfer analysis in MHD flow of Casson fluid over a vertical plate embedded in a porous medium with arbitrary wall shear stress. Journal of Porous Media. 2018; 21(8), 739-748.

10. VeeraKrishna M, Subba Reddy G, Chamka AJ. Hall effects on unsteady MHD oscillatory free convective flow of second grade fluid through porous medium between two vertical plates. AIP Physics of Fluids. 2018; 30(2), 1.

11. Krishna Prasad DVV, Krishna Chaitanya GS, Srinivasa Raju R. Role of Casson fluid on MHD natural convection flow towards vertically inclined plate with hall current. AIP Conference Proceedings. 2018; 1953(1), 1400731-1400734.

12. Amanulla CH, Nagendra N, Subba Rao A, Beg OA, Kadir A. Numerical exploration of thermal radiation and biot number effects on the flow of a non-Newtonian MHD Williamson fluid over a vertical convective surface. Heat Transfer Asian Research. 2018; 47(2), 286-304.

13. Veera Krishna M, Kamboji Jyothi, Ali J. Chamkha. Heat and mass transfer on unsteady, MHD oscillatory flow of second grade fluid through a porous medium between two vertical plates, under the influence of fluctuating heat source/sink, and chemical reaction. International Journal Fluid Mechanics Research. 2018; 45(8), 459-477. 
14. Lakshmanna B, Venkateswarlu S. Unsteady MHD convective flow of an incompressible viscous fluid through porous medium over a vertical plate. Annals of Pure and Applied Mathematics. 2018; 16(2), 451-460.

15. Janardhana Reddy G, Kethireddy B, Kumar M, Mainul Hoque M. A molecular dynamics study on transient non-Newtonian MHD Casson fluid flow dispersion over a radiative vertical cylinder with entropy heat generation. Journal of Molecular Liquids. 2018; 252, 245-262.

16. Rajakumar KVB, Balamurugan KS, Ramana Murthy V. Radiation absorption and viscous dissipation effects on MHD free convective flow past a semi infinite moving vertical porous plate. International Journal of Fluid Mechanics Research. 2018; 45(5), 439-458.

17. Ashwinkumar GP, Sulochana C, Samrat SP. Effect of the aligned magnetic field on the boundary layer analysis of magnetic nanofluid over a semi infinite vertical plate with ferrous nanoparticles. Multidiscipline Modeling in Materials and Structures. 2018; 14(3), 497-515.

18. Ganesh Kumar K, Archana M, Gireesha BJ, Krishna Murthy MR, Rudraswamy NG. Cross diffusion effect on MHD mixed convection flow of nonlinear radiative heat and mass transfer of Casson fluid over a vertical plate. Results in Physics. 2018; 8, 694-701.

19. Hari Krishna Y, Venkata Ramana Reddy G, Makinde OD. Chemical reaction effect on MHD flow of Casson fluid with porous stretching sheet. Defect and Diffusion Forum. 2018; 389, 100-109. 